\title{
El fenómeno youtuber y el uso de la lengua vasca en el ámbito educativo The youtuber phenomenon and the use of the basque language in education
}

\author{
Aintzane Etxebarria ${ }^{1}$, Aitor Iglesias ${ }^{1}$, Urtza Garay ${ }^{2}$ \\ Aintzane.etxebarria@ehu.eus, aitor.iglesias@ehu.eus,urtza.garay@ehu.eus \\ ${ }^{1}$ Didáctica de la Lengua y la Literatura \\ UPV/EHU \\ Bilbao, España \\ 2 Didáctica y Organización Escolar \\ UPV/EHU \\ Bilbao, España
}

\begin{abstract}
Resumen- En este trabajo se presenta el proyecto docente "Youtuber lehiaketa" (Concurso Youtuber), llevado a cabo en la Facultad de Educación de Bilbao. Este proyecto se ha desarrollado en el campo de la comunicación digital y la educación usando las TRI, Tecnologías de la Relación, la Información y la Comunicación, y ha tenido como objetivo fomentar el uso de una lengua minoritaria como el euskera entre su alumnado y así poder desarrollar la competencia comunicativa oral. En el ha participado alumnado de tres asignaturas diferentes de manera activa, dinámica, plural y cooperativa. Los resultados obtenidos muestran que la herramienta digital YouTube ha servido para aumentar el uso de la lengua vasca entre las personas que han participado en este proyecto.
\end{abstract}

Palabras clave: aprendizaje cooperativo, youtuber, euskera, comunicación digital

Abstract- This work presents the teaching project "YouTuber lehiaketa" (YouTuber contest), carried out at the Faculty of Education of Bilbao. This project has been developed in the field of digital communication and education using RICTs, Relation, Information and Communication Technologies, and has aimed to promote the use of a minority language such as Basque among its students and thus be able to develop oral communication skills. Students from three different subjects have participated in an active, dynamic, plural and cooperative way. The results obtained show that the digital tool YouTube has served to increase the use of the Basque language among the people who have participated in this project.

Keywords: cooperative learning, youtuber, basque language, digital communication

\section{INTRODUCCIÓN}

En el campo de la comunicación digital y la educación, numerosos estudios se han centrado en las Tecnologías de la Relación, la Información y la Comunicación (TRIC) (Gabelas, et. al., 2012; Linares, et. al., 2019; Bernal, et. al., 2019) y se han definido como "espacios de conversación, juego, recreación, interacción y construcción; risas y cotilleos; parodias y flirteos, los que generan un conjunto de oportunidades para aprender las denominadas habilidades para la vida, en su capacidad para sentir y emocionarse, socializarse y conocer" (Gabelas et. al.,
2012). La plataforma YouTube es un espacio que convierte a las personas comunes en comunicadores influyentes:

"For the first time in history, common people becoming communicators with great influence, gatekeepers or non-official agents who determine the message flow to their community (Scolari, 2013; Jenkins et al., 2015) thanks to their use of YouTube platform and their social networks." (Blanco \& Sainz de Baranda 2018)

A nivel mundial, YouTube registra unos 2,000 millones de usuarios al mes, se considera el servicio de contenido en video más consultado, quedando por encima de Netflix e Instagram (https://www.forbes.com.mx/). Según las estadísticas sobre YouTube que se pueden consultar en la Red, de media al mes, 8 de cada 10 personas de 18 a 49 años ven YouTube, más que la televisión. Se puede navegar en unos 80 idiomas diferentes en 91 países (https://www.brandwatch.com/es/blog/46estadisticas-youtube). Tal y como se deduce de estos datos, el alcance de este medio digital es enorme.

El Youtuber o usuario que realiza un vídeo se convierte en comunicador social, y debe saber de qué hablar, para quién, cuándo, dónde, y en qué forma, por lo tanto, debe mostrar una serie de características propias de la competencia comunicativa (Hymes, 1971) para ser entendido.

\section{CONTEXTO}

En la Comunidad Autónoma del País Vasco, se hablan numerosas lenguas que nos llegan de diferentes partes del mundo, pero hay dos lenguas oficiales que son el euskera y el castellano. Cada cinco años, se realiza una Encuesta Sociolingüística a la población mayor de 15 años con el objetivo de obtener información sobre el conocimiento, uso y transmisión familiar del euskera. Los datos de la última VI Encuesta Sociolingüística (2016), nos dicen que el 33,9\% de la población de 16 o más años que vive en la CAE es vascohablante, pero los bilingües equilibrados que se expresan con la misma fluidez tanto en euskera como en castellano, son solamente el 29,3\% de los vascohablantes. Además, los bilingües que se desenvuelven mejor en castellano que en 
euskera son el $44 \%$, y constituyen el colectivo mayoritario entre los vascohablantes, más de la mitad de los vascohablantes de entre 16-24 años son bilingües con predominio del castellano $(55,7 \%)$. En este contexto, el alumnado de la Facultad de Educación que será futuro docente con edades comprendidas entre 19 y 22 años debe desarrollar la competencia comunicativa oral del euskera porque entre otras razones en la Agenda Euskadi 2030 (https://www.euskadi.eus/contenidos/informacion/agenda2030/ es_def/adjuntos/agenda_multinivel.pdf) se establecen como metas para el desarrollo sostenible el impulso del plurilingüismo en la escuela para el desarrollo de proyectos lingüísticos y para la euskaldunización del ámbito escolar; además de la promoción de medidas de política lingüística necesarias para promocionar el uso del euskera en todos los ámbitos de la vida social y familiar.

En una investigación realizada por Arroyabe y col. (2020) sobre la presencia del euskera en el consumo y creación de contenidos audiovisuales en línea llegan a la conclusión de que la presencia de esta lengua en contenidos audiovisuales en línea entre los adolescentes de la Comunidad Autónoma Vasca es escasa, y al igual que Paricio-Martín y col. (2010) defienden la creación de recursos audiovisuales online para revitalizar o promover una lengua minoritaria:

Las experiencias que se han aplicado y estudiado en lenguas minorizadas de todo el mundo han obtenido resultados que en la mayoría de los casos invitan al optimismo. Basta repasar la bibliografía sobre revitalización de lenguas como el hawaiano, el maorí, el galés, el irlandés, el mohawk, el occitano, el sardo, el amhárico, etc. (Wright, 2004; Galla, 2009; Cunliffe et al., 2005; Eisenlohr, 2004; Bittinger, 2006; Djordjevic', 2007; Streiter et al., 2006), para observar cómo, en las lenguas cuyas comunidades tienen acceso a las nuevas tecnologías, estas desempeñan un papel muy relevante en los programas de revitalización que se están desarrollando, bien de forma planificada - como en el caso del hawaiano desde 1994- (Galla, 2009), bien a partir de la acción individual de activistas o hablantes, como en el caso del occitano (Djordjevic', 2007). (Paricio-Martín \& Martínez-Cortés, 2010: 2)

Por lo tanto, el proyecto que presentamos tiene como objetivo trabajar la competencia comunicativa oral a través del fenómeno youtuber de los jóvenes estudiantes de la facultad y responder a la siguiente cuestión: ¿Contribuye el fenómeno youtuber a un mayor uso de la lengua oral vasca en el ámbito educativo no formal, y más específicamente dentro del grupo de trabajo?

\section{DESCRIPCIÓN}

A continuación, se describe el proyecto docente, el perfil del alumnado de primer curso que ha llevado a cabo el proyecto en cuanto a las costumbres lingüísticas, la repercusión de este proyecto en el uso de la lengua vasca dentro del grupo de trabajo y los recursos que se han empleado para su medición y los principales resultados.

\section{A. Participantes en el concurso Youtuber}

Con el fin de impulsar el uso de la lengua vasca entre amigos, fuera y dentro de espacios académicos, se llevó a cabo la creación de un vídeo YouTuber. Para ello se preparó un proyecto basado en el Aprendizaje Cooperativo y Dinámico, que pone su acento en que los estudiantes sean los dueños de su aprendizaje y sean formados de forma integral, flexible y adaptada a las necesidades de la sociedad (https:/www.ehu.eus/es/web/sae-helaz/ikd-curriculumgarapena-oinarriak).
En cuanto al desarrollo del proyecto, el alumnado debió convertirse en "euskal YouTuber" y fomentar el uso del euskera en ámbitos educativos fuera del aula. Se crearon pequeños grupos que tuvieron ayuda y acompañamiento de grupos de estudiantes de cursos superiores; por un lado, les guiaron en los aspectos didáctico formales que debe mantener un buen YouTuber educativo y en la investigación sociolingüística previa a la elaboración del vídeo; por otro lado, les aportaron datos sobre la historia y el uso del euskera a lo largo de la historia. De esta manera, en el Aprendizaje Cooperativo y Dinámico de los y las estudiantes se sigue la Metodología Basada en Proyectos Colaborativos Interniveles mediante la cooperación intensiva del alumnado de los diferentes niveles educativos y la metodología basada en la investigación, puesto que el alumnado realizó una investigación que se basaba en la medición del uso de las lenguas guiada por el profesorado implicado y en colaboración con una entidad experta en este tipo de investigaciones

Antes de comenzar con el proyecto docente y para conocer el perfil de los estudiantes que tomaron parte en el mismo, se diseñó una encuesta sobre sus costumbres lingüísticas, debían responder si principalmente se comunicaban en castellano, en euskera o en ambas lenguas parecido en los siguientes ámbitos: en la cafetería, en el aula, en el campus, en las peceras y por medio de WhatsApp con los compañeros, con los bedeles en la Facultad, con el personal administrativo de secretaría, con el personal de la biblioteca, con el equipo decanal en el Decanato. De este análisis se observa que los datos obtenidos en el ámbito académico y fuera de él, según la última encuesta sociolingüística (2016) no varían mucho, puesto que el principal idioma de comunicación entre los jóvenes es el castellano (tabla $1)$. 
Tabla1. Datos sobre la lengua que utilizan los jóvenes para comunicarse entre ellos en las distintas zonas del ámbito académico.

\begin{tabular}{|c|c|c|c|c|}
\hline \multicolumn{5}{|c|}{ CON LOS BEDELES } \\
\hline & Frecuencia & Porcentaje & $\begin{array}{c}\text { Porcentaje } \\
\text { válido }\end{array}$ & $\begin{array}{c}\text { Porcentaje } \\
\text { acumulado }\end{array}$ \\
\hline $\begin{array}{c}\text { Parecido en } \\
\text { ambas } \\
\text { lenguas }\end{array}$ & 9 & 11,3 & 11,3 & 11,3 \\
\hline $\begin{array}{c}\text { Casi } \\
\text { siempre o } \\
\text { siempre en } \\
\text { euskera } \\
\end{array}$ & 67 & 83,8 & 83,8 & 95,0 \\
\hline $\begin{array}{c}\text { Casi } \\
\text { siempre o } \\
\text { siempre en } \\
\text { castellano }\end{array}$ & 4 & 5,0 & 5,0 & 100,0 \\
\hline Total & 80 & 100,0 & 100,0 & \\
\hline \multicolumn{5}{|c|}{$\begin{array}{c}\text { CON EL } \\
\text { PERSONAL } \\
\text { DE LA BIBLIOTEC }\end{array}$} \\
\hline $\begin{array}{c}\text { Parecido en } \\
\text { ambas } \\
\text { lenguas }\end{array}$ & 26 & 32,5 & 32,5 & 32,5 \\
\hline $\begin{array}{c}\text { Casi } \\
\text { siempre o } \\
\text { siempre en } \\
\text { euskera } \\
\end{array}$ & 28 & 35,0 & 35,0 & 67,5 \\
\hline $\begin{array}{c}\text { Casi } \\
\text { siempre o } \\
\text { siempre en } \\
\text { castellano }\end{array}$ & 26 & 32,5 & 32,5 & 100,0 \\
\hline Total & 80 & 100,0 & 100,0 & \\
\hline \multicolumn{5}{|c|}{$\begin{array}{c}\text { CON EL } \\
\text { PERSONAL } \\
\text { DMINISTRATIVO }\end{array}$} \\
\hline $\begin{array}{c}\text { Parecido en } \\
\text { ambas } \\
\text { lenguas }\end{array}$ & 7 & 8,8 & 8,8 & 8,8 \\
\hline $\begin{array}{c}\text { Casi } \\
\text { siempre o } \\
\text { siempre en } \\
\text { euskera } \\
\end{array}$ & 71 & 88,8 & 88,8 & 97,5 \\
\hline $\begin{array}{c}\text { Casi } \\
\text { siempre o } \\
\text { siempre en } \\
\text { castellano }\end{array}$ & 2 & 2,5 & 2,5 & 100,0 \\
\hline Total & 80 & 100,0 & 100,0 & \\
\hline \multicolumn{5}{|c|}{$\begin{array}{c}\text { CON EL } \\
\text { EQUIPO } \\
\text { DECANAL } \\
\end{array}$} \\
\hline $\begin{array}{c}\text { Parecido en } \\
\text { ambas } \\
\text { lenguas }\end{array}$ & 6 & 7,5 & 7,5 & 7,5 \\
\hline $\begin{array}{c}\text { Casi } \\
\text { siempre o } \\
\text { siempre en } \\
\text { euskera } \\
\end{array}$ & 73 & 91,3 & 91,3 & 98,8 \\
\hline $\begin{array}{c}\text { Casi } \\
\text { siempre o } \\
\text { siempre en } \\
\text { castellano } \\
\end{array}$ & 1 & 1,3 & 1,3 & 100,0 \\
\hline Total & 80 & 100,0 & 100,0 & \\
\hline
\end{tabular}

En cambio, con el personal docente y administrativo casi siempre o siempre se comunican en euskera, salvo en la biblioteca (tabla2)
Tabla 2. Datos sobre la lengua que utilizan los jóvenes para comunicarse con personal docente, administrativo y en la biblioteca.

\begin{tabular}{|c|c|c|c|c|}
\hline \multicolumn{5}{|c|}{$\begin{array}{c}\text { EN LA } \\
\text { CAFETERÍA }\end{array}$} \\
\hline & Frecuencia & Porcentaje & $\begin{array}{c}\begin{array}{c}\text { Porcentaje } \\
\text { válido }\end{array} \\
\end{array}$ & $\begin{array}{l}\text { Porcentaje } \\
\text { acumulado }\end{array}$ \\
\hline $\begin{array}{c}\text { Parecido en } \\
\text { ambas lenguas }\end{array}$ & 10 & 12,5 & 12,5 & 12,5 \\
\hline $\begin{array}{l}\text { Casi siempre o } \\
\text { siempre en } \\
\text { euskera }\end{array}$ & 6 & 7,5 & 7,5 & 20,0 \\
\hline $\begin{array}{l}\text { Casi siempre o } \\
\text { siempre en } \\
\text { castellano }\end{array}$ & 64 & 80,0 & 80,0 & 100,0 \\
\hline Total & 80 & 100,0 & 100,0 & \\
\hline \multicolumn{5}{|c|}{ EN LA AULA } \\
\hline $\begin{array}{c}\text { Parecido en } \\
\text { ambas lenguas }\end{array}$ & 22 & 27,5 & 27,5 & 27,5 \\
\hline $\begin{array}{l}\text { Casi siempre o } \\
\text { siempre en } \\
\text { euskera } \\
\end{array}$ & 10 & 12,5 & 12,5 & 40,0 \\
\hline $\begin{array}{l}\text { Casi siempre o } \\
\text { siempre en } \\
\text { castellano }\end{array}$ & 48 & 60,0 & 60,0 & 100,0 \\
\hline Total & 80 & 100,0 & 100,0 & \\
\hline \multicolumn{5}{|c|}{ SEL CAMPUS } \\
\hline $\begin{array}{c}\text { Parecido en } \\
\text { ambas lenguas }\end{array}$ & 10 & 12,5 & 12,5 & 12,5 \\
\hline $\begin{array}{c}\text { Casi siempre o } \\
\text { siempre en } \\
\text { euskera } \\
\end{array}$ & 5 & 6,3 & 6,3 & 18,8 \\
\hline $\begin{array}{l}\text { Casi siempre o } \\
\text { siempre en } \\
\text { castellano }\end{array}$ & 65 & 81,3 & 81,3 & 100,0 \\
\hline Total & 80 & 100,0 & 100,0 & \\
\hline \multicolumn{5}{|c|}{ IR WHATSAPP } \\
\hline $\begin{array}{c}\text { Parecido en } \\
\text { ambas lenguas }\end{array}$ & 15 & 18,8 & 18,8 & 18,8 \\
\hline $\begin{array}{l}\text { Casi siempre o } \\
\text { siempre en } \\
\text { euskera }\end{array}$ & 7 & 8,8 & 8,8 & 27,5 \\
\hline $\begin{array}{l}\text { Casi siempre o } \\
\text { siempre en } \\
\text { castellano }\end{array}$ & 58 & 72,5 & 72,5 & 100,0 \\
\hline Total & 80 & 100,0 & 100,0 & \\
\hline \multicolumn{5}{|c|}{$\begin{array}{l}\text { EN LAS } \\
\text { PECERAS }\end{array}$} \\
\hline $\begin{array}{c}\text { Parecido en } \\
\text { ambas lenguas }\end{array}$ & 40 & 50,0 & 50,0 & 50,0 \\
\hline $\begin{array}{c}\text { Casi siempre o } \\
\text { siempre en } \\
\text { euskera }\end{array}$ & 12 & 15,0 & 15,0 & 65,0 \\
\hline $\begin{array}{l}\text { Casi siempre o } \\
\text { siempre en } \\
\text { castellano }\end{array}$ & 28 & 35,0 & 35,0 & 100,0 \\
\hline Total & 80 & 100,0 & 100,0 & \\
\hline
\end{tabular}




\section{B. Proyecto docente}

En el Grado de Educación Primaria en el primer curso se trabaja la asignatura básica de rama en euskera Desarrollo de la Competencia Comunicativa: Lengua Castellana y Lengua Vasca en Educación Primaria I. Esta asignatura se imparte según el modelo educativo IKD (Aprendizaje Cooperativo y Dinámico). Es un modelo propio, cooperativo, plurilingüe e inclusivo en el que los estudiantes trabajan de manera activa, dinámica, plural y cooperativa (https://www.ehu.eus/es/web/sae-helaz/ikd-curriculumgarapena-oinarriak) con el objetivo de llevar a cabo los diferentes proyectos que se les plantean.

En la asignatura titulada Desarrollo de la Competencia Comunicativa: Lengua Castellana y Lengua Vasca en Educación Primaria I se pidió al alumnado de primer curso del año académico 2019/2020 que se presente a un concurso de youtubers. Estos estudiantes estaban tutorizados por otros de cuarto curso que estaban matriculados en la asignatura Lenguas e innovación en la Escuela y en la Lenguas y Sociedad en el País Vasco. El objetivo más importante que se intentó conseguir a través de este proyecto educativo durante diez semanas fue el siguiente: fomentar el uso del euskera en ámbitos educativos no formales, más específicamente dentro del grupo de trabajo por medio de youtube; para ello, el alumnado de primero debía convertirse en "euskal youtuber". Además de trabajar en las aulas de primer curso la competencia comunicativa oral no formal con el profesor de manera teórica y práctica, se crearon pequeños grupos que tenían ayuda y acompañamiento de grupos de estudiantes de $4^{\circ}$ curso, que les guiaron en los aspectos didácticos formales que debía mantener un buen youtuber educativo (asignatura Lenguas e innovación en la Escuela), y les aportaron nociones teóricas que debían aparecer en el vídeo sobre el uso y conocimiento de esta lengua en la sociedad vasca (asignatura Lenguas y Sociedad en el País Vasco). La cooperación entre los grupos de estudiantes dio como resultado el video estilo youtuber que presentaron al concurso público para toda la Facultad.

\section{RESUltados}

Para saber si la inclusión de la tecnología para el fomento del uso del euskera por medio del uso intensivo de la red social Youtube había contribuido a potenciar el uso del euskera se aplicó una prueba sobre las costumbres lingüísticas de los estudiantes antes y después del proyecto, esta prueba consistía en señalar el idioma en el que hablaban con cada uno de los integrantes del grupo cooperativo, según la siguiente escala: 1) sólo en euskera, 2) mayoritariamente en euskera, 3) mayoritariamente en castellano, 4) sólo en castellano.

Partiendo de los datos obtenidos se han comparado las medias de las muestras antes y después del proyecto y se observa que aumenta el uso de la lengua minoritaria (tabla 3 ).

Tabla 3: Comparación de medias en el uso de la lengua minoritaria antes y después del proyecto educativo.

\begin{tabular}{|l|r|r|r|r|}
\hline & $\mathrm{N}$ & \multicolumn{1}{|c|}{ Media } & $\begin{array}{c}\text { Desviación } \\
\text { estándar }\end{array}$ & $\begin{array}{c}\text { Media de } \\
\text { error } \\
\text { estándar }\end{array}$ \\
\hline Antes del proyecto & 80 & 3,225 &, 7459 &, 0834 \\
Después del proyecto & 80 & 2,425 &, 6940 &, 0776 \\
\hline
\end{tabular}

\section{CONCLUSIONES}

Teniendo en cuenta el contexto sociolingüístico de la mayoría del alumnado que acude a las aulas del Grado de Educación Primaria, se pensó en un proyecto para fomentar el uso de una lengua minoritaria que convive con otra que se utiliza más tanto en el ámbito privado como en el público, incluido el académico.

Este proyecto tiene como referencia el modelo educativo IKD cuyo principio es el aprendizaje cooperativo y dinámico, y en este caso se pensó en la utilización de YouTube con el objetivo principal de impulsar la lengua vasca en dos direcciones: 1) en los grupos de trabajo de los Youtubers y 2) en Internet, puesto que los estudiantes que querían tenían opción de compartir sus vídeos.

Los resultados muestran que el proyecto y más concretamente la herramienta digital YouTube ha servido para aumentar el uso de la lengua vasca entre los integrantes del grupo de trabajo.

Esto nos lleva a pensar que Internet puede ser una potente herramienta para la normalización lingüística de aquellas lenguas que tienen un uso limitado, bien como una plataforma de difusión de contenido, bien como un recurso de comunicación social. Queda por saber si esta herramienta ayuda a mejorar la competencia comunicativa del alumnado en cuanto a las propias estructuras lingüísticas de la lengua.

\section{Agradecimientos}

Este trabajo es el resultado del proyecto i320-34 que ha impulsado el Vicerrectorado de Innovación, Responsabilidad Social y Acción Cultural de la UPV/EHU.

\section{REFERENCIAS}

Bases para el desarrollo curricular de las titulaciones oficiales de la UPV/EHU. (2021, julio 19). Recuperado de https://www.ehu.eus/es/web/sae-helaz/ikd-curriculumgarapena-oinarriak

Bernal, L., Gabelas, J. A. \& Marta, C. (2019). Las tecnologías de la relación, la información y la comunicación (TRIC) como entorno de integración social. Interface (Botucatu), 23.

Blanco, M. \& Sainz de Baranda, C. (2018). Channels produced by LGBT+ YouTubers: gender discourse analysis. Observatorio (OBS*) Special issue on the co-option of audiences in the attention economy, 97-121.

Fernandez de Arroyabe, A., Eguskiza, L. \& Lazkano, I. (2020). El futuro de las lenguas minoritarias en Internet en manos de los jóvenes prosumidores. El caso vasco. Cuadernos.info, 46, 367-396.

Gabelas, J. A., Marta, C. \& Aranda, D. (2012). Por qué las TRIC y no las TIC. COMeIN, 9.

Gobierno Vasco / Eusko Jaurlaritza (2016). VI Encuesta sociolingüística 2016, Servicio Central de Publicaciones / Argitalpen Zerbitzu Nagusia.

Hymes, D. H. (1971). On communicative competence. Philadelphia: University of Pennsylvania.

Linares, E., Aristegui, I. \& Beloki, U. (2019). YouTube, una plataforma para la (in)formación, relación, comunicación, 
diversión, y gestión de indentidades (de género) en la natividad digital. Revista Mediterránea de Comunicación / Mediterranean Journal of Communication, 10(1), 55-70.
Paricio-Martín, S.; Martínez-Cortés, J.P. (2010). Nuevas vías de revitalización para lenguas minorizadas: la repercusión de in-ternet en el caso del aragonés. Digithum, 12, 1-11. 